\title{
Predischarge Checklist
}

The discharging physician must control his/her prescriptions and deal with all the following items before patient's discharge (check each box).

EKG acquisition

DAPT prescription

Explanation of DAPT risks and benefits

Follow-up visit scheduling,

Explanation of Heart Failure management (with scheduled follow-up visit to Heart Failure center if indicated)

Explanation of Diabetes management (with scheduled follow-up visit to Diabetes center if indicated)

Explanation of lifestyle adjustments

Discharging Physician Signature. 